\title{
Out-of-equilibrium dynamics of the Hopfield model in its spin-glass phase
}

\author{
Marcelo A. Montemurro, ${ }^{1}$ Francisco A. Tamarit, ${ }^{1}$ Daniel A. Stariolo, ${ }^{2}$ and Sergio A. Cannas ${ }^{1}$ \\ ${ }^{1}$ Facultad de Matemática, Astronomía y Física, Universidad Nacional de Córdoba, Ciudad Universitaria, 5000 Córdoba, Argentina \\ ${ }^{2}$ Instituto de Física, Universidade Federal do Rio Grande do Sul, Caixa Postal 15051, 91501-970 Porto Alegre, Brazil
}

(Received 29 February 2000)

\begin{abstract}
In this paper, we study numerically the out-of-equilibrium dynamics of the Hopfield model for associative memory inside its spin-glass phase. Aside from its interest as a neural network model, it can also be considered as a prototype of a fully connected magnetic system with randomness and frustration. By adjusting the ratio between the number of stored configurations $p$ and the total number of neurons $N$, one can control the phase-space structure, whose complexity can vary between the simple mean-field ferromagnet (when $p=1$ ) and that of the Sherrington Kirkpatrick spin-glass model (for a properly taken limit of an infinite number of patterns). In particular, little attention has been devoted to the spin-glass phase of this model. In this paper, we analyze the two-time autocorrelation function, the decay of the magnetization and the distribution of overlaps between states. The results show that within the spin-glass phase of the model, the dynamics exhibits aging phenomena and presents features that suggest a non trivial breaking of replica symmetry.
\end{abstract}

PACS number(s): 75.10.Nr, 64.60.Ht

In recent years, the off-equilibrium dynamics of spin glasses below the freezing temperature has been the subject of a great number of studies in the field of complex magnetic systems [1], both experimental and theoretical. Real spin glasses are characterized by such an extremely slow dynamics that they may never attain equilibrium within experimental time scales. Under these circumstances, a theoretical description of the actual physics of real spin glasses requires a dynamical approach.

Statistical physics models, despite their simplifications, have shown to be very useful in the understanding of the behavior of these materials. Among them, perhaps the most relevant one in the development of this subject, is the wellknown Edwards-Anderson (EA) model. Since a complete analytical description of this model has not been achieved up to now (due to the enormous mathematical difficulties involved), numerical simulations emerged as the main tool of research in this area. However, in order to implement them, it is necessary to provide the system with an adequate dynamics, usually accomplished by means of a stochastic (Monte Carlo) process. Although these ad hoc dynamics were originally introduced to compute equilibrium quantities, quite surprisingly they have also proved to be very useful in simulating the actual dynamical processes observed in real materials [2]. This agreement opened up a whole new range of possibilities in statistical physics research by allowing physicists to simulate, with simple models and Monte Carlo dynamics, the complex out-of-equilibrium behavior of spin glasses and other magnetic materials.

Concerning equilibrium properties, the long-range version of the EA model due to Sherington and Kirkpatrick [3] (SK model), has raised particular interest owing to the fact that an exact solution is known for its thermostatics [4] and insightful approximations have been found even for its dynamics [5]. The picture that emerges for this model is that of a phase space with a very intricate hierarchical structure of basins, whose number and depth diverge with the size of the system-as depicted by the solution due to Parisi [4].
Moreover, within each of those basins, which divide the system into independent ergodic components, there is as well a complex structure of subvalleys within subvalleys separated by barriers with a continuous distribution of heights. Within the framework of this complicated phase-space geometry, the ensuing dynamics turns out to be of an extremely slow character and a wide range of new time-dependent phenomena are observed, which collectively are referred to as aging phenomena. Starting from random initial conditions, such a system may never achieve true equilibrium; therefore, its inherent physics must be interpreted as dynamical in nature. Whether this picture is shared by low-dimensional systems or not, is still under enduring discussions.

The Hopfield model of neural networks has been thoroughly studied in connection with its static and dynamical properties of retrieval, which together determine its usefulness as an associative memory model. Aside from its value as a neural model, it is possible to look upon the Hopfield model in the broader context of complex magnetic systems. In this sense, we can consider it as another kind of longrange spin-glass model with a different choice of coupling distribution, and with the added advantage of having a phase-space structure whose complexity can be controlled. Both static and dynamical studies of the Hopfield model have concentrated mainly on the retrieval phase and close to the basin of attraction of a stored memory pattern; consequently, in such circumstances, the very rich spin-glass structure underlying the free-energy landscape has received little attention so far.

The thermostatics of the Hopfield model has been completely solved assuming that replica symmetry holds. While this seems to be the correct solution within the retrieval zone (except for very low temperatures [6]), it has not been obvious until now, as far as we know, which symmetry-breaking scheme yields the correct solution within the spin-glass phase of the model.

In particular, some corrections, due to symmetry-breaking effects, have been found at very low temperatures [7]; nonetheless, it has always been sustained that the influence on the 
retrieval capabilities of the network, induced by a breaking in replica symmetry, do not have any noticeable effect on the retrieval performance of the system [6].

The main objective of this paper is twofold. First, it aims to get insight into the underlying structure of the spin-glass phase of the Hopfield model. This will eventually help to find the correct Ansatz to solve its thermodynamics. On the other hand, since it is possible to control the richness of its phase-space structure, ranging from a simple ferromagnet, when only one pattern is stored, to the SK at the other extreme for a properly taken limit of an infinite number of patterns [8], it will allow us to extract important conclusions concerning the influence of such structure on the offequilibrium dynamics of magnetic systems.

The outline of this paper is as follows: first we describe the model we used and the methods of our simulations. Then we present the results and their interpretation within the framework of long-range spin glasses, and finally we discuss our conclusions and suggest further paths of research on the subject.

\section{MODEL}

The Hopfield model of neural networks is described by the following Hamiltonian [10]:

$$
H[J]=-\frac{1}{2} \sum_{i j} J_{i j} S_{i} S_{j},
$$

where the sum goes over all the possible pairs $i, j$. The variable $S_{i}$ represents the state of the $i$ th Ising spin (neuron) at the discrete time $t$, and $J_{i j}$ is the coupling constant between the $i$ th and the $j$ th neurons. The coupling matrix $J$ is built according to Hebb's rule in order to store a definite set of $p$ randomly chosen configurations (patterns):

$$
J_{i j}=\left\{\begin{array}{l}
\frac{1}{N} \sum_{\mu=1}^{p} \xi_{i}^{\mu} \xi_{j}^{\mu} \quad \text { if } i \neq j \\
0 \quad \text { if } i=j,
\end{array}\right.
$$

where we denote each of the $p$ patterns as $\vec{\xi}^{\mu}=\left\{\xi_{i}^{\mu}\right\}$ ( $\mu$ $=1, \ldots, p, i=1, \ldots, N$, where $N$ is the total number of spins).

The time evolution of the model is governed by a standard heat-bath Monte Carlo process with sequential update, and we say that it performs as an associative memory system if each of the $p$ stored patterns is close enough to an attractor of the dynamics. In fact, when this happens, the structure of the phase space is fairly complex, as a huge number of other attractors also appear alongside [11]. The simplest of them are the reverse states, i.e., the actual patterns with all the spins inverted. Other states that are stable points of the dynamics are the spurious attractors (mixture states) and the spin-glass states. A complete solution of the model can be found in [6] and we reproduce in Fig. 1 the phase diagram obtained therein.

The labeled curves delineate the borders of qualitatively differentiated regimes of the Hopfield model in the $T-\alpha$ plane, where $\alpha=p / N$. All over the roughly triangular sector enclosed by the $T_{M}$ line, the stored patterns are close to

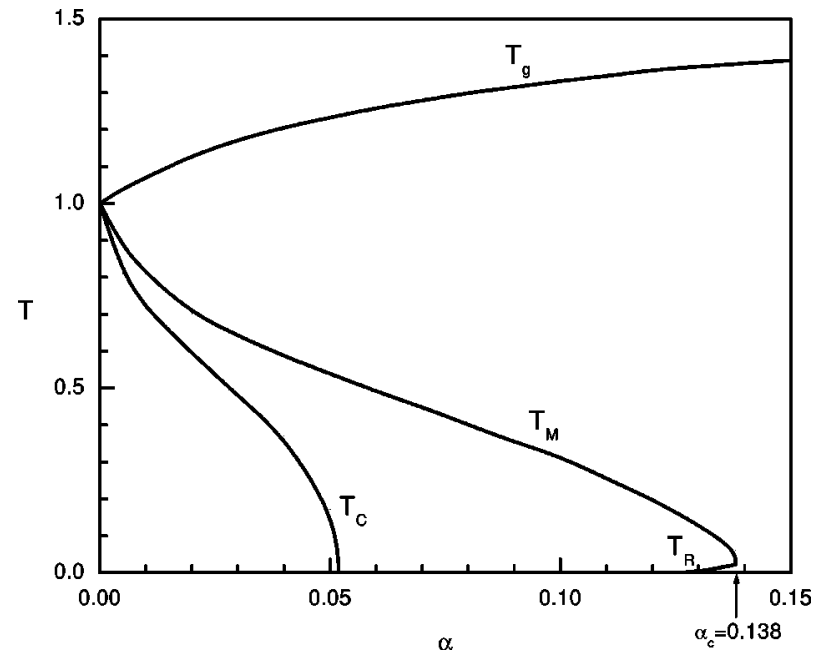

FIG. 1. Phase diagram for the Hopfield model. See the main text for a brief explanation. After Amit et al. [6].

dynamical attractors, therefore the system performs well as an associative memory device. Still, we need to discriminate between two distinct zones associated with the retrieval states that differ in their phase-space structure. Inside the region below the $T_{c}$ line, the retrieval attractors represent absolute minima of the free energy. A different structure is found in the region between the $T_{c}$ and the $T_{M}$ lines where the global minima correspond to spin-glass states totally uncorrelated with the patterns. However, the retrieval attractors still stand as local minima of the free energy, thus allowing the system to work as an associative memory as long as the dynamics is initiated close enough to a retrieval basin. Then, as the $T_{M}$ line is crossed, the retrieval states disappear abruptly and the spin-glass states become the only ones present. This spin-glass phase covers all the region beyond the $T_{M}$ line and below $T_{g}$-the latter having a simple mathematical expression, namely: $T_{g}=1+\sqrt{\alpha}$. Finally, above $T_{g}$ all the free-energy structure is lost as the system enters a paramagnetic phase. The line labeled $T_{R}$ shows the limit of validity of the replica-symmetry ansatz.

We performed simulations on systems with a number of spins ranging between 128 and 4000, with particular emphasis in $N=500$ and $N=1000$. Throughout this work, the time is measured in units of whole Monte Carlo sweeps over the network of $N$ spins.

\section{RESULTS}

In this section, we present and describe the results of our simulations for a range of values of the parameter $\alpha$, covering the spin-glass phase of the Hopfield model.

\section{A. Aging}

In attempting to characterize the dynamics of the model, we first carried out some numerical experiments focused on revealing the presence of slow dynamics in conjunction with history-dependent phenomena, i.e., aging. These features are most easily found numerically in simulations concerning the two-time autocorrelation function $C\left(t, t^{\prime}\right)$, which turns out to show a strong explicit dependence on both times over a wide range of time scales. For a system that has achieved equilib- 
rium, it is expected that $C\left(t, t^{\prime}\right)$ is homogeneous in time, depending on $t$ and $t^{\prime}$ only through their difference. However, in spin-glass systems in their glassy phase exhibiting aging phenomena, a much more complicated behavior arises that reveals the basis for the scenario that has been called weak ergodicity breaking (WEB) [13]. In the realm of longrange spin glasses, the onset of slow dynamics and historydependent phenomena below a definite transition temperature is normally associated with a highly complex freeenergy surface with a plethora of metastable states. In a system evolving through an energy landscape so complex as that found in long-range spin glasses, the many basins within basins may play the role of dynamical traps that, having a continuous distribution of heights without bounds, can thereby confine the system in such a way that the average escape time goes to infinity.

We can write down the basic features of the WEB scenario more explicitly, regarding the behavior observed in the two-time autocorrelation function, as follows:

$$
\frac{\partial C\left(t_{w}, t+t_{w}\right)}{\partial t} \leqslant 0 \quad \frac{\partial C\left(t_{w}, t+t_{w}\right)}{\partial t_{w}} \geqslant 0,
$$

$$
\lim _{t \rightarrow \infty} C\left(t_{w}, t+t_{w}\right)=0 \quad \forall \text { fixed } t_{w},
$$

where $t_{w}$ indicates the time that the system has been left to evolve after a sudden quench from infinite temperature to a low temperature state, and $t$ is the time measured since the waiting time $t_{w}$.

In all our simulations we chose a set of $p$ random patterns, where each of the $\xi_{i}^{\mu}$,s is taken from the following distribution:

$$
P\left(\xi_{i}^{\mu}\right)=\frac{1}{2}\left[\delta\left(\xi_{i}^{\mu}-1\right)+\delta\left(\xi_{i}^{\mu}+1\right)\right] .
$$

In order to take measurements of the two-time autocorrelation function, we computed numerically the following expression:

$$
C\left(t_{w}, t+t_{w}\right)=\left[\frac{1}{N} \sum_{i=1}^{N} S_{i}\left(t_{w}\right) S_{i}\left(t+t_{w}\right)\right]_{a v},
$$

where we have denoted by $[\cdots]_{a v}$ an average taken over several realizations of the random patterns and thermal baths. The abrupt quench from infinite temperature to the glassy phase was emulated by always starting our simulations from random initial configurations.

In many occasions in this paper we shall refer to the SK model for comparison, and consequently we have included in Fig. 2 the results of a simulation carried out on that modelwhich although performed by us for this work, it is a result that had already been reported in the literature [14]. This figure corresponds to a system with $N=1000$ spins and with couplings taken from a Gaussian distribution normalized so that $T_{g}=1$. In this case the temperature of the thermal bath was chosen as $T=0.4 T_{g}$. The graph shows $C\left(t_{w}, t+t_{w}\right)$ vs $t$ for different waiting times ranging from $t_{w}=2$ to $t_{w}=8192$.

The first result for the Hopfield model is shown in Fig. 3,

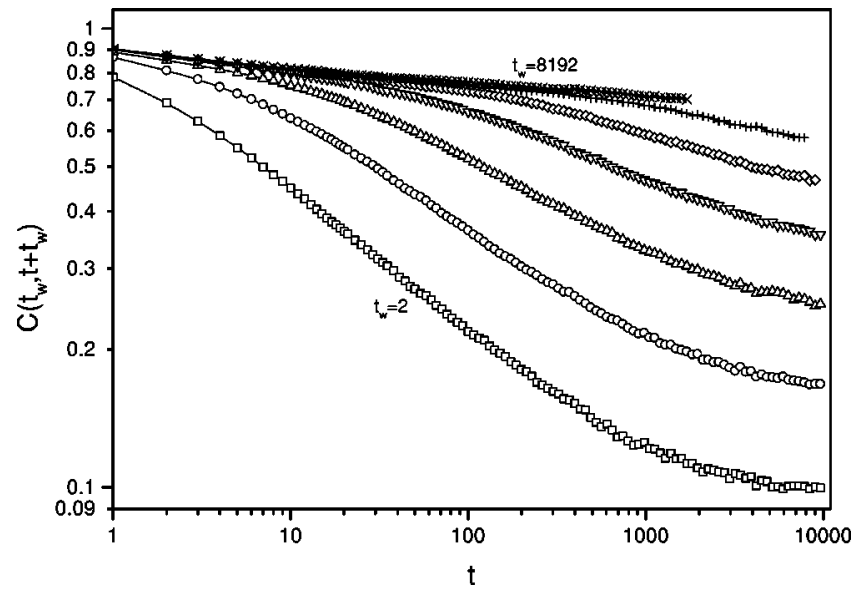

FIG. 2. Two-time autocorrelation functions $C\left(t_{w}, t+t_{w}\right)$ vs $t$ for the SK model, with $N=1000$, at $T=0.4 T_{g}$ and with couplings taken from a Gaussian distribution. The waiting times are 2, 8, 32, 128, $512,2048,8192$. The exhibited data are an average over 400 realizations of the couplings, initial conditions and thermal baths.

and it pertains to a system with $N=1000, \alpha=1$, and $T$ $=0.4 T_{g}$; inside the spin-glass phase and far enough from the $T_{M}$ line to avoid strong size effects. The behavior of the autocorrelation function confirms clearly the presence of aging in the Hopfield model in this region, in agreement with the WEB picture described by Eq. (5). The curves show an explicit dependence on both times indicating that equilibrium has not been attained within the time of the simulation. Furthermore, its decay becomes slower for longer waiting times as it is expected within the context of aging phenomena. It is worth noticing that the qualitative features of the graph are roughly similar to those found in the SK model, in particular for the longest waiting times.

From the standpoint of this result, it is expected that similar features of aging are found for larger values of $\alpha$, since we know that for $\alpha \rightarrow \infty$ the Hopfield model converges to the SK model. A more delicate point to be concerned with refers to the behavior of the Hopfield model in regions of its spin-

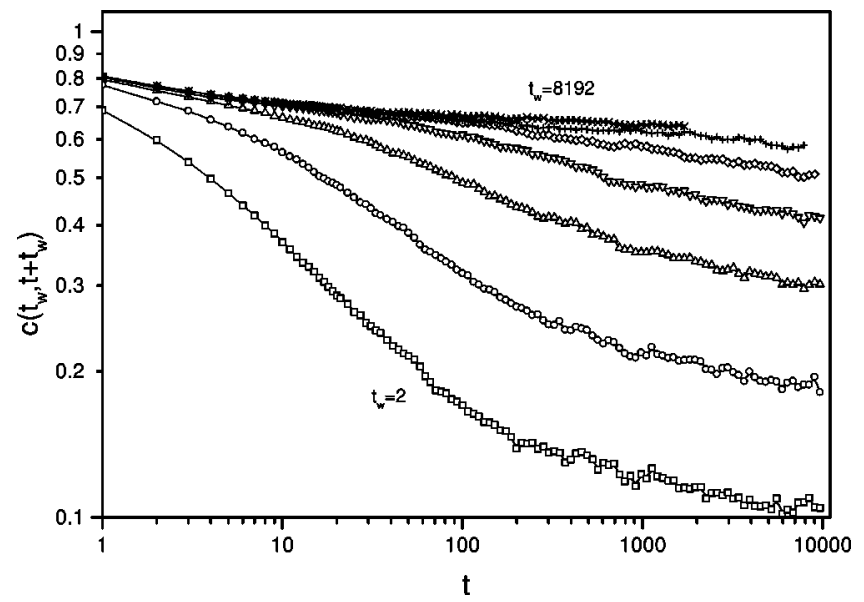

FIG. 3. Two-time autocorrelation functions $C\left(t_{w}, t+t_{w}\right)$ vs $t$ for the Hopfield model, with $N=1000$, at $T=0.4 T_{g}$ and $\alpha=1$. The waiting times are $2,8,32,128,512,2048,8192$. The exhibited data are an average over 200 realizations of the set of memory patterns, initial conditions and thermal baths. 


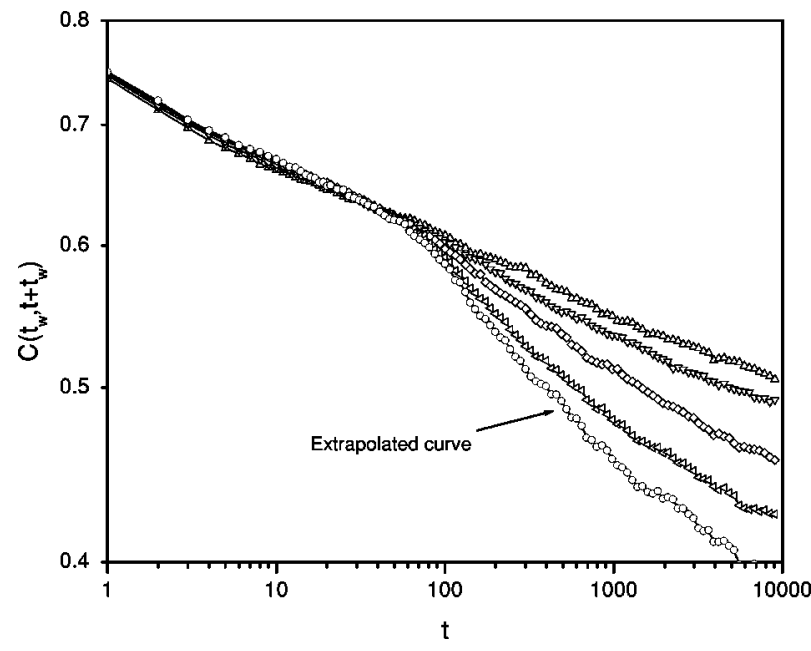

FIG. 4. Two-time autocorrelation functions $C\left(t_{w}, t+t_{w}\right)$ vs $t$ for the Hopfield model, with $t_{w}=128$, at $T=0.4 T_{g}, \alpha=0.2$ and different sizes $(N=500,1000,2000$, and 4000 from top downward). The lowest curve (full circles) corresponds to the extrapolated values.

glass phase closer to the retrieval zone. To address that issue, we studied the dynamics of the model for a value of $\alpha$ $=0.2$, which is close to the retrieval zone and at the same time lies at a safe distance from the critical $T_{M}$ transition line, whose exact location shifts toward larger values of $\alpha$ due to finiteness of the system. Besides, we had to deal with other manifestation of finite-size effects, in this case related to the less degreee of frustration in the couplings as the value of $\alpha$ is lowered. It is worth stressing that this strong dependence on $N$ was not observed for larger values of $\alpha$ (see below). This can be easily understood in the following terms: higher values of $\alpha$ imply more frustration in the couplings, and so higher energy barriers between metastable states. For small values of $\alpha$ the system can then quickly thermalize, and the time it requires for thermalizing depends strongly on $N$. On the other hand, for large values of $\alpha$ the sizes of the energy barriers make the thermalizing time increase far beyond our simulation times and therefore we observe nonequilibrium phenomena that do not depend on the size of the system.

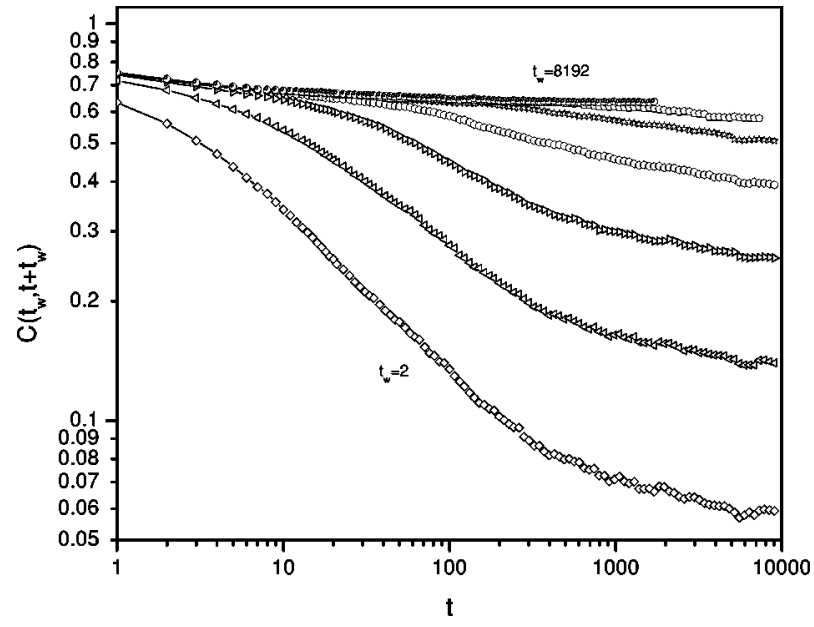

FIG. 5. Extrapolated two-time autocorrelation functions $C\left(t_{w}, t+t_{w}\right)$ vs $t$ for the Hopfield model, at $T=0.4 T_{g}$ and $\alpha=0.2$. The rest of the parameters are the same as those of Fig. 2.

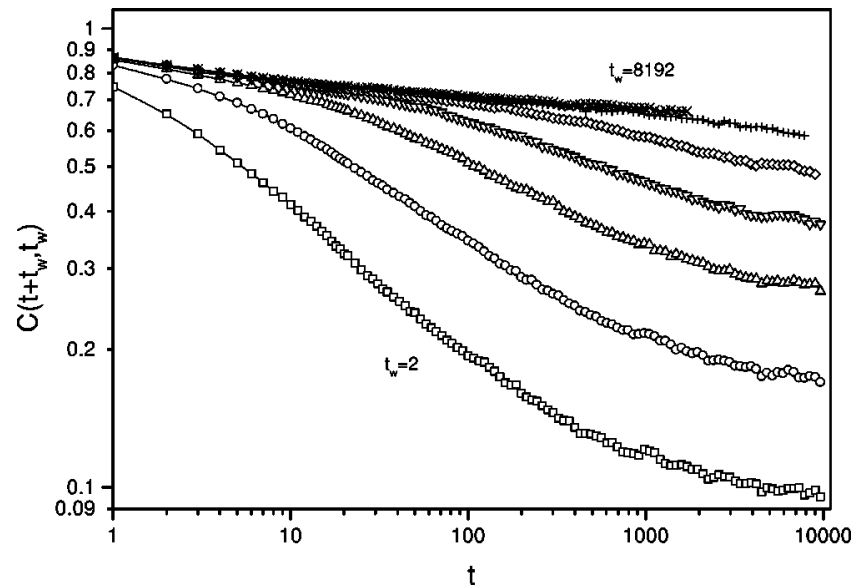

FIG. 6. Two-time autocorrelation functions $C\left(t_{w}, t+t_{w}\right)$ vs $t$ for the Hopfield model, with $N=1000$, at $T=0.4 T_{g}$ and $\alpha=10$. The rest of the parameters are the same as those of Fig. 2.

To analyze the finite-size effects, we carried out simulations with system sizes ranging from $N=500$ to $N=4000$. In Fig. 4 we show $C\left(t_{w}, t+t_{w}\right)$ vs $t$ for $t_{w}=128$ and $N=500$, 1000, 2000, 4000 (open symbols from top downward), where we see that even for the largest size we considered the dependence on $N$ is still strong. Since larger systems are beyond our computational capacity we decided to extrapolate the data to $N \rightarrow \infty$, assuming that the $C_{N}\left(t_{w}, t+t_{w}\right)$ varies smoothly with $1 / N$, and keeping terms up to second order. The lowest curve (full symbols) in Fig. 4 corresponds to the extrapolated values obtained for $t_{w}=128$. In Fig. 5 we plot all the extrapolated curves $C\left(t_{w}, t+t_{w}\right)$ vs $t$.

In Figs. 6 and 7 we show the results for $\alpha=10$ and $\alpha$ $=100$ (with $N=1000$ and $N=500$, respectively, since as $\alpha$ increases the results have less dependence on $N$ ). Let us observe that the behavior shown by the Hopfield model in this range of $\alpha$ values is much like that of the SK model.

Another quite relevant issue is finding an appropriate scaling law for the aging curves. As has already been pointed out in the literature $[5,12]$, systems that exhibit a continuous distribution of relaxation times, like the SK, may not obey a simple scaling relation. Instead, for systems with a unique

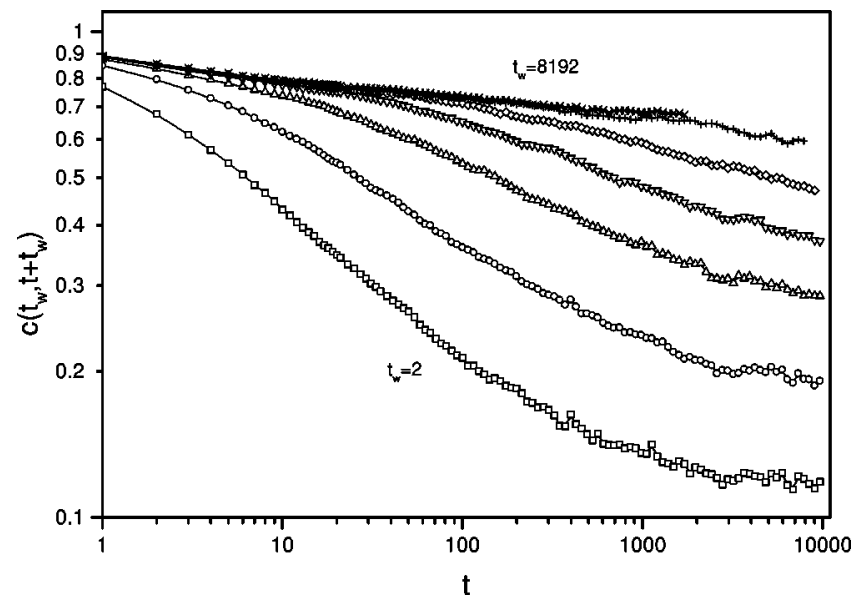

FIG. 7. Two-time autocorrelation functions $C\left(t_{w}, t+t_{w}\right)$ vs $t$ for the Hopfield model, with $N=500$, at $T=0.4 T_{g}$ and $\alpha=100$. The rest of the parameters are the same as those of Fig. 2. 


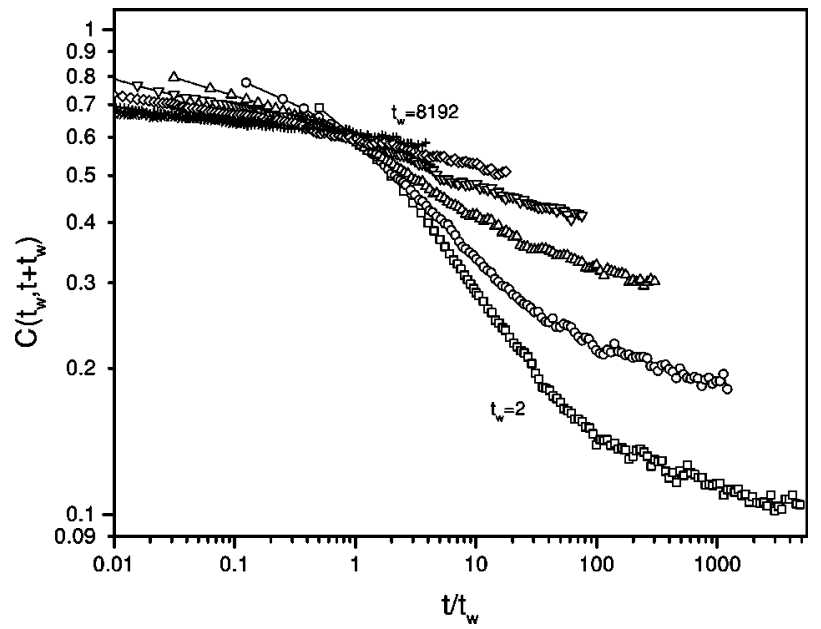

FIG. 8. Two-time autocorrelation functions $C\left(t_{w}, t+t_{w}\right)$ vs $t / t_{w}$ for the Hopfield model, with $N=1000$, at $T=0.4 T_{g}$ and $\alpha=1$. The rest of the parameters are the same as those of Fig. 2 .

relevant time scale (for instance, the $p$-spin model) it is possible to find simple laws that cause all the aging curves to collapse into a single one. As an example, let us put forward the simplest of those scalings, usually called naive scaling, which takes the following form [9]:

$$
C\left(t_{w}, t+t_{w}\right)=f\left(\frac{t}{t_{w}}\right) .
$$

A likewise dependence is observed in other models that can be well described by theories in which full replica symmetry holds [9]. The graph in Fig. 8 shows $C\left(t_{w}, t+t_{w}\right)$ vs $t / t_{w}$ for the data displayed in Fig. $3(\alpha=1)$, and brings the Hopfield model even closer to the SK, as it depicts exactly the same patterns found in that model [12]. That is, on the one hand the roughly common crossing point at $t / t_{w}$ (with a corresponding value of approximately $1-T / T_{g}$ on the autocorrelation axis); on the other, the dependence shown by $C\left(t_{w}, t\right.$ $+t_{w}$ ) for increasing values of $t_{w}$ when $\tau \equiv t / t_{w}$ is kept fixed: it increases when $\tau>1$, whereas it decreases for $\tau<1$.

The observed lack of agreement between the scaled curves serves as an indication of the complexity of the many time scales involved-as was already mentioned in connection with the SK model. Next we tried the following more complex expression, which had already been used on the SK model yielding a better scaling [12]:

$$
C\left(t_{w}, t+t_{w}\right)=f\left(\frac{\ln \left(t+t_{w}\right)}{\ln \left(t_{w}\right)}\right) .
$$

In Figs. 9-11, we have plotted $C\left(t_{w}, t+t_{w}\right)$ vs $\ln (t$ $\left.+t_{w}\right) / \ln \left(t_{w}\right)$ for $\alpha=0.2,1$ and 10, respectively (the curves for $\alpha=0.2$ were obtained from the extrapolated values displayed in Fig. 5). By analyzing the graphs, we notice that the data fall into two different groups, depending on the particular age (waiting time) of the system. In the first place, the curves for $t_{w}=2$ and $t_{w}=8$, which are associated to a very young system, do not show a good scaling in any of the figures. On the other hand, the data corresponding to all the other (longer) waiting times are appreciably better scaled by relation (7). As regards Fig. 11, it is worth noticing that the

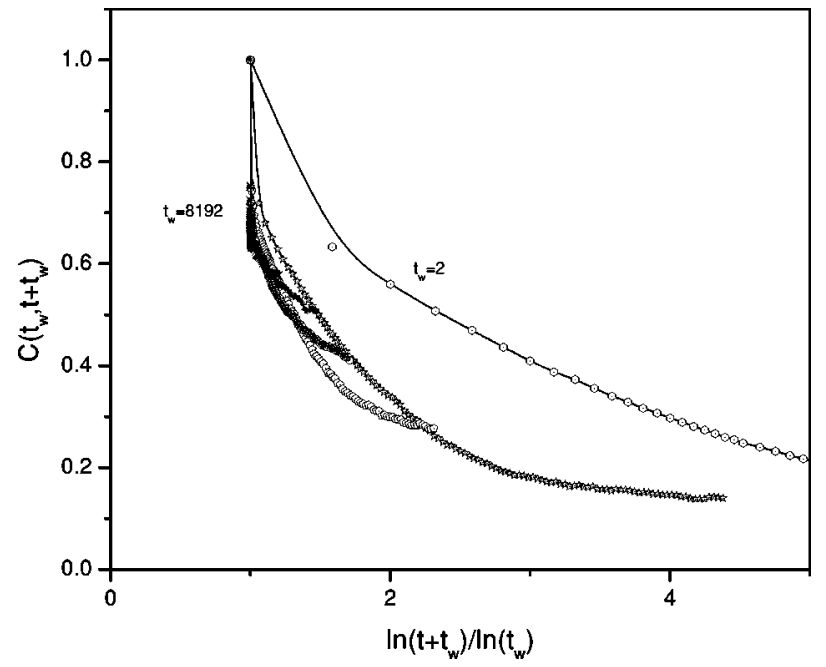

FIG. 9. Extrapolated two-time autocorrelation functions $C\left(t_{w}, t+t_{w}\right)$ vs $\ln \left(t+t_{w}\right) / \ln \left(t_{w}\right)$ for the Hopfield model, at $T$ $=0.4 T_{g}$ and $\alpha=0.2$. The rest of the parameters are the same as those of Fig. 2.

graph bears a remarkable resemblance to the one obtained for the SK model in reference [12] using the same scaling expression 7. This also applies to the little departures from a correct superposition observed when $t \gg 1$. Thus, we can conclude that in the spin-glass phase we do not find any critical value of $\alpha$ (at least larger than 0.2) below which the replica-symmetry ansatz holds. On the contrary, our evidence suggests that the spin-glass phase has a phase-space structure very similar to that observed in the SherringtonKirkpatrick model.

\section{B. Decay of the magnetization}

In systems that possess a huge number of metastable states in the presence of quenched disorder, like the SK, the decay to equilibrium of physical quantities usually obeys power laws. We ran a series of simulations in which we started the Hopfield model from a totally magnetized state,

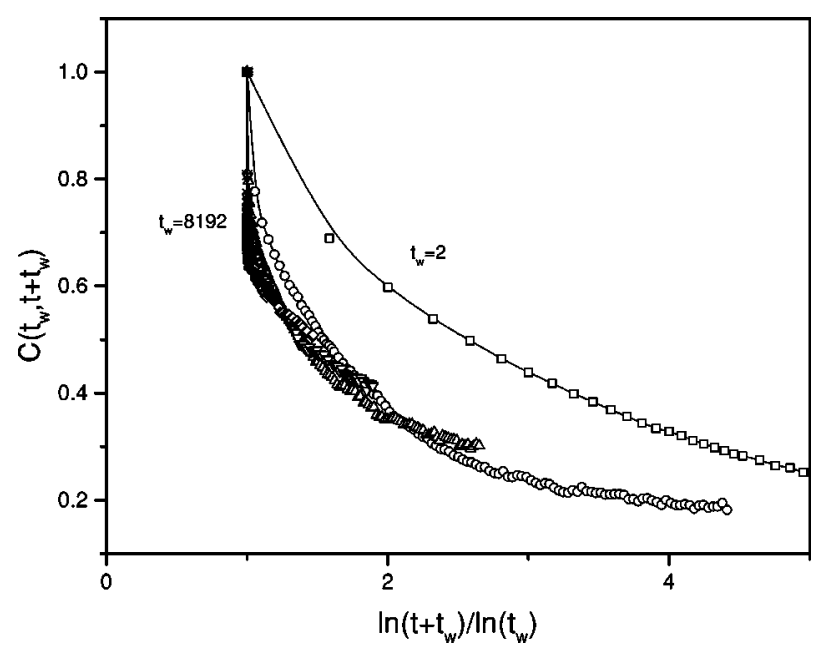

FIG. 10. Two-time autocorrelation functions $C\left(t_{w}, t+t_{w}\right)$ vs $\ln \left(t+t_{w}\right) / \ln \left(t_{w}\right)$ for the Hopfield model, with $N=1000$, at $T=0.4 T_{g}$ and $\alpha=1$. The rest of the parameters are the same as those of Fig. 2. 


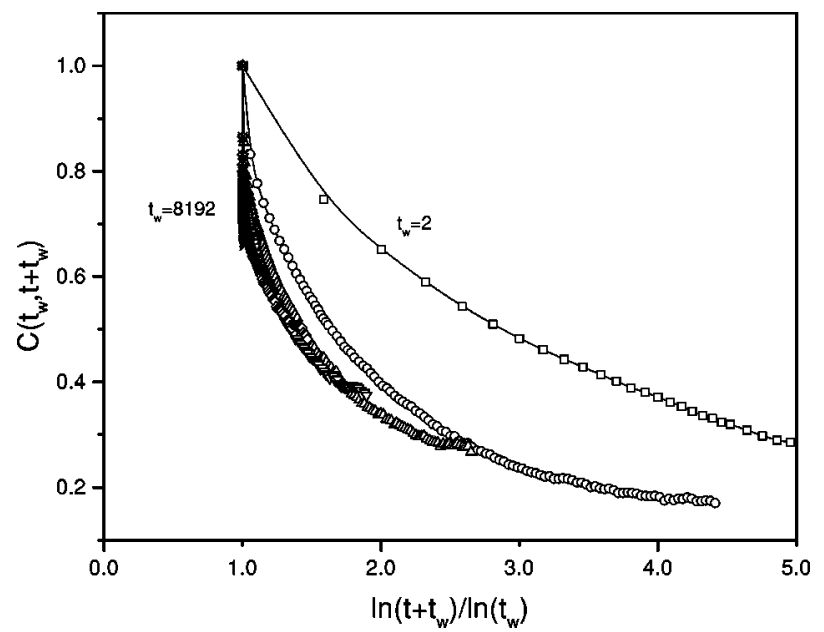

FIG. 11. Two-time autocorrelation functions $C\left(t_{w}, t+t_{w}\right)$ vs $\ln \left(t+t_{w}\right) / \ln \left(t_{w}\right)$ for the Hopfield model, with $N=1000$, at $T=0.4 T_{g}$ and $\alpha=10$. The rest of the parameters are the same as those of Fig. 2.

i.e., $S_{i}(0)=1 \forall i$, and let it relax in contact with a thermal bath and no external fields applied. In this case we monitored the quantity

$$
m(t)=\left[\frac{1}{N} \sum_{i} S_{i}(t)\right]_{a v} .
$$

Then we fitted the obtained data with the same expression used in [12]:

$$
m(t)=m_{\infty}+\tilde{m} t^{-\delta(T)} .
$$

The results of the simulations are presented in Fig. 12, where we have plotted $\delta(T)$ vs $T$ for a system with $N$ $=500$ and $\alpha=1$. The exponent turns out to be a linear function of temperature in a striking agreement with the behavior shown by the SK model [12].

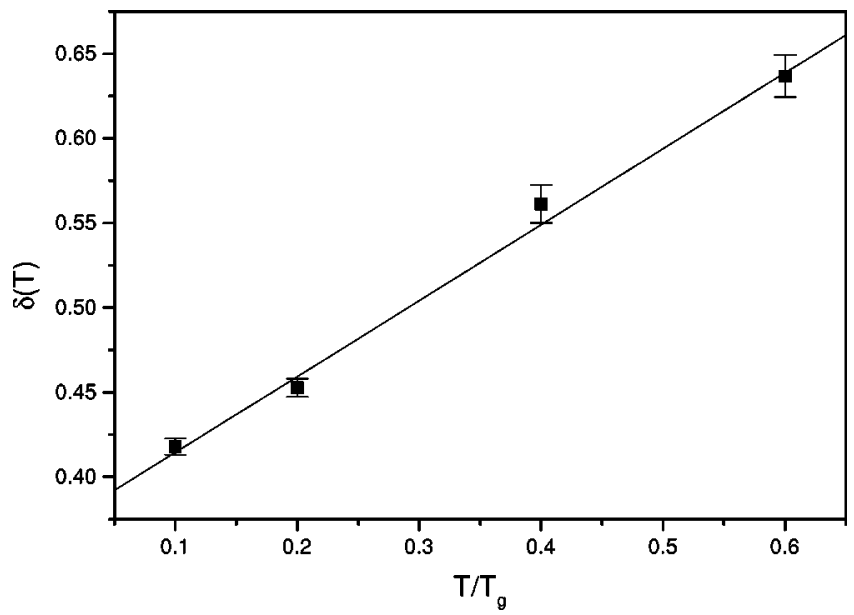

FIG. 12. Magnetization-decay exponent $\delta(T)$ vs $T / T_{g}$. The data corresponds to a system with $N=500$ and $\alpha=1$.

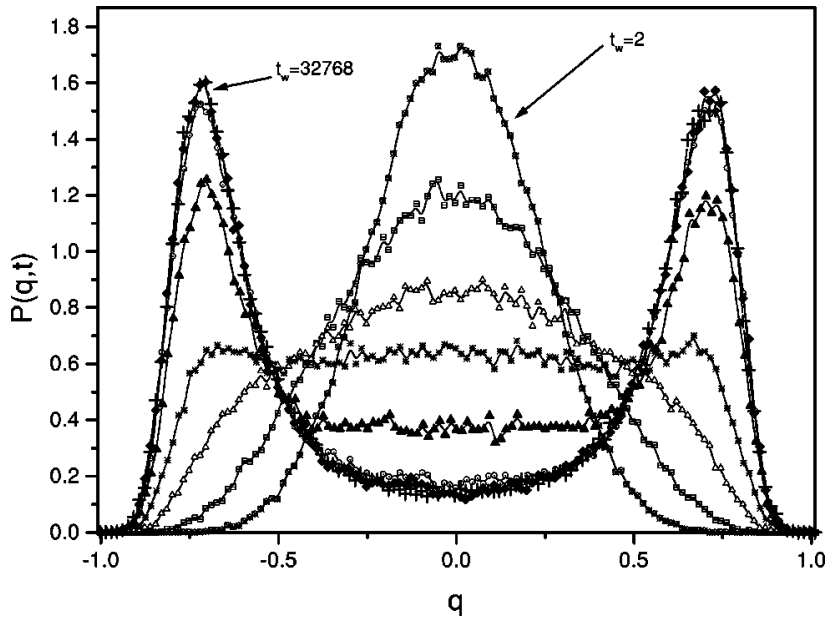

FIG. 13. Overlap distribution $P(q, t)$ vs $q$, for a system with $N=128$ and $\alpha=1$. The different curves correspond to the following times: $2,4,8,16,64,512,4096,32768$. A total of 15 replicas were used in each of 744 runs with different realizations of the random patterns and thermal noise.

\section{Distribution of overlaps}

The overlap distribution contains much information regarding the geometric structure of phase space, and its asymptotic form may unveil a complex arrangement of many metastable states. We performed a numeric computation of the time-dependent overlap distribution $P(q, t)$. To that purpose we started randomly a set of 15 replicas of the system and let them evolve with independent thermal baths and no couplings between them; at prescribed times we took measurements of all the possible overlaps between the replicas, and repeated this procedure for several realizations of the set of random patterns. Finally, we made a histogram with all the gathered data.

As it evolves, each system shall probe different regions of the complex free-energy landscape, searching stochastically for basins of increasing depth. Hence, their mutual overlaps - at large times - are expected to render some insight into the deeper structure of phase space. The corresponding overlaps between each pair of replicas were evaluated according to the following expression:

$$
q^{\alpha, \beta}(t)=\frac{1}{N} \sum_{i=1}^{N} S_{i}^{\alpha}(t) S_{i}^{\beta}(t),
$$

where the superscripts denote different replicas. The results of this simulation can be seen in Fig. 13 and correspond to a system size of $N=128$ spins and $T=0.4 T g$. As we started the replicas from random initial conditions, for short times the distributions must be approximately Gaussian. Subsequently, the nearly Gaussian shape distorts by broadening at the base, reaching an almost uniform distribution for $t=16$ (stars in the graph). From that time on the curve two clearly defined peaks begin to develop, which grow in height and sharpen while converging asymptotically to the final shape for long times. It is also interesting to notice that the data converge rapidly to a limiting value for $q=0$ that is different from zero. From the above mentioned facts we conclude that the distribution for $t \rightarrow \infty$ consists of two Dirac $\delta$ functions 
(corresponding to $\pm q_{E A}$ ) plus a continuous distribution between them. This is the same result that has been obtained both numerically [14] and analytically for the SK model.

The Edwards-Anderson order parameter stands as a geometric indicator that roughly establishes the size of the deepest basins visited by the system in its evolution. The usual way of estimating its value numerically is by measuring the position of the two peaks in the distribution $P(q, t)$ for $t$ $\rightarrow \infty$. Hence, from the data of our simulations we obtain approximately $q_{E A}^{(H o p)} \approx 0.73$. Furthermore, we have tested this result against the value predicted by the replicasymmetric solution of the Hopfield model by solving numerically the saddle-point equations of [6]; the calculations yielded $\bar{q}_{E A}^{(\text {Hop })}=0.6167$, which differs considerably from the measured one and in fact falls quite close to the value obtained for the SK model within the replica-symmetric approximation [3]. These results may be compared with the value of the order parameter predicted by Parisi's solution of the SK model, which is given with good approximation by the following expression [15]:

$$
q_{E A}^{(S K)}=1-\frac{3}{2}\left(\frac{T}{T_{g}}\right)^{2} .
$$

For $T / T_{g}=0.4$, Eq. (11) yields $q_{E A}^{(S K)}=0.76$, in good agreement with our data for the Hopfield model. The slight discrepancy might be due to an $\alpha$ dependence in the Hopfield model jointly with size effects.

These facts altogether support strongly what has already been suggested by our aging simulations on the Hopfield model - that the dynamics for long times is essentially that of the SK model.

\section{CONCLUSIONS}

In our simulations we have analyzed the glassy dynamics of the Hopfield model in the intermediate region between its retrieval phase and the SK limit for $\alpha \rightarrow \infty$. So far, no analytical solution has been found for the model's dynamics that incorporates a full breaking of replica symmetry; therefore, our results may shed some light in the asymptotic behavior that ought to be required in analytical treatments of the problem.

The first and foremost conclusion is that the Hopfield model in its spin-glass phase-for intermediate values of $\alpha$-shows a complex dynamics that fits neatly into the scenario of weak ergodicity breaking. Comparing the two-time autocorrelation curves found for the Hopfield and SK models, we conclude that for long times, both systems behave similarly in the whole range of $\alpha$ 's we studied. The numerical evidence we collected supports the conclusion that the phase-space structure of the Hopfield model is much more complex than that expected for a long-range magnetic system in which replica symmetry holds. Likewise, the failing in trying to collapse all the aging curves into a single one by means of a simple scaling law may as well indicate the presence of a continuous range of temporal scales in the dynamics.

In the intermediate region on the $\alpha$ scale the decay of the magnetization was found to obey a power law, indicating the existence of a great number of metastable states. In addition, the decay exponent showed a linear dependence on temperature, notably like that observed in the SK model. This last fact let us go a step further and suggest a very similar organization of the vast collection of metastable states in the two models.

The characteristics of the overlap distribution at long times contribute some important facts regarding the structure of the most profound basins in the free-energy hypersurface. On one side we noticed the evidence of a continuous portion in the distribution, and on the other, the Edwards-Anderson order parameter that we obtained for the Hopfield modelfor $\alpha=1$ and $T=0.4 T_{g}$-is very much close to the theoretical value found analytically from Parisi's theory for the SK model. Once more, these facts point at an inherent resemblance of the two models under comparison.

Summing up the numerical results altogether, we have arrived at the following picture for the Hopfield model in the studied range of parameters: in the spin-glass phase the freeenergy landscape of the Hopfield model differs from that of the SK model mainly in the regions farthest away from the deepest basins, which are precisely the ones that a randomly started configuration is very much likely to visit first. Nevertheless, as the system evolves and diffuses into the rugged high-dimensional free-energy surface it encounters a geometric structure that is essentially the same as for the SK model.

Among the extensions of the present paper, we mention that there are ongoing studies aimed at determining the nature of the $\alpha$ dependence of the phase-space structure in the retrieval phase below the $T_{M}$ transition line, where the simultaneous presence of different types of attractors may lead to the emergence of complex aging behavior.

\section{ACKNOWLEDGMENTS}

This work was partially supported by grants from Consejo Nacional de Investigaciones Científicas y Técnicas CONICET (Argentina), Consejo Provincial de Investigaciones Científicas y Tecnológicas CONICOR (Córdoba, Argentina), and Secretaría de Ciencia y Tecnología de la Universidad Nacional de Córdoba SECYT (Córdoba, Argentina). D.A.S. was partially supported by $\mathrm{CNPq}$ (Brazil).
[1] J.P. Bouochaud, L. F. Cugliandolo, J. Kurchan, and M. Mézard, in Spin Glasses and Random Fields, edited by. A.P. Young (World Scientific, Singapore, 1997), p. 161.

[2] Z.B. Li, U. Ritschel, and B. Zheng, J. Phys. A 27, L837 (1994).
[3] D. Sherrington and S. Kirkpatrick, Phys. Rev. Lett. 35, 1972 (1975).

[4] G. Parisi, J. Phys. A 13, L371 (1980).

[5] L. Cugliandolo and J. Kurchan, J. Phys. A 27, 5749 (1994).

[6] D. Amit, H. Gutfreund, and H. Sompolinsky, Ann. Phys. 
(N.Y.) 173, 30 (1987).

[7] A. Crisanti, D. Amit, and H. Gutfreund, Europhys. Lett. 2, 337 (1986).

[8] F. Benamira, J. Provost, and G. Valee, J. Phys. (Paris) 46, 1269 (1985).

[9] A. Bray, Adv. Phys. 43, 357 (1994).

[10] J.J. Hopfield, Proc. Natl. Acad. Sci. U.S.A. 79, 2554 (1982).

[11] D. Amit, H. Gutfreund, and H. Sompolinsky, Phys. Rev. A 32,
1007 (1985).

[12] E. Marinari, G. Parisi, and D. Rossetti, Eur. Phys. J. B 2, 495 (1998).

[13] J.P. Bouchaud, J. Phys. I 2, 1705 (1992).

[14] H. Takayama, H. Yoshino, and K. Hukushima, J. Phys. A 30, 3891 (1997).

[15] K. Binder and A.P. Young, Rev. Mod. Phys. 58, 801 (1986). 